220 Proceedings of the National Academy of Sciences of Belarus. Physical-technical series, 2021, vol. 66, no. 2, pp. 220-226

ISSN 1561-8358 (Print)

ISSN 2524-244X (Online)

УДК 66.096.5:536.24

https://doi.org/10.29235/1561-8358-2021-66-2-220-226

Поступила в редакцию 12.03.2021

Received 12.03.2021

\author{
Э.К. Бучилко \\ Институт тепло- и массообмена имени А. В. Лыкова Национальной академии наук Беларуси, Минск, \\ Республика Беларусь

\section{ВНЕШНИЙ ТЕПЛООБМЕН В ПСЕВДООЖИЖЕННОМ КВАЗИБИДИСПЕРСНОМ ЗЕРНИСТОМ СЛОЕ}

\begin{abstract}
Аннотация. Приведены результаты экспериментального исследования внешнего теплообмена с трубным пучком в псевдоожиженном бидисперсном слое с выраженной бимодальностью распределения частиц по размерам. Установлены зависимость коэффициента теплоотдачи от скорости фильтрации воздуха, влияние массовой доли крупных частиц в смеси на максимальный коэффициент теплоотдачи. Получена интерполяционная зависимость для расчета оптимальной скорости фильтрации исследуемых смесей. В качестве эквивалентного диаметра при расчете теплоотдачи рекомендовано использовать среднеповерхностный диаметр частиц полидисперсной смеси. Разработана методика расчета максимального коэффициента теплоотдачи и оптимальной скорости фильтрации для квазибидисперсного кипящего слоя. Метод основан на использовании коэффициентов теплоотдачи и оптимальных скоростей фильтрации отдельных составляющих бидисперсной смеси. Получены уравнения для расчета максимального коэффициента теплоотдачи и оптимальной скорости фильтрации в бидисперсной смеси.

Ключевые слова: теплообмен, кипящий слой, бидисперсный слой, эквивалентный диаметр, максимальный коэффициент теплоотдачи, оптимальная скорость фильтрации

Для цитирования. Бучилко, Э.К. Внешний теплообмен в псевдоожиженном квазибидисперсном зернистом слое / Э. К. Бучилко // Вес. Нац. акад. навук Беларусі. Сер. фіз.-тэхн. навук. - 2021. - Т. 66, №2. - С. $220-226$. https://doi.org/10.29235/1561-8358-2021-66-2-220-226
\end{abstract}

Eduard K. Buchilko

A.V. Luikov Heat and Mass Transfer Institute of the National Academy of Sciences of Belarus, Minsk, Republic of Belarus.

\title{
EXTERNAL HEAT-EXCHANGE IN A QUASI-BIDISPERSE GRANULAR FLUIDIZED BED
}

Abstract. This paper presents the results of an experimental study of the external heat-exchange with a tube bundle in a bidisperse fluidized bed with marked bimodality of particle size distribution. The dependence of the maximum heat-transfer coefficient on the rate of air filtration was determined. The influence of the mass fraction of large particles on the heat-transfer coefficient in a mixture has been established. An interpolation dependence for calculating the optimal filtration rate of the investigated mixtures has been obtained. It is recommended to use the average surface diameter of the polydisperse particles mixture as the equivalent diameter when calculating the heat-exchange. A method for calculating the maximum heat-transfer coefficient and the optimal filtration rate for a quasi-bidisperse fluidized bed has been developed. The method is based on the use of heat transfer coefficients and optimal filtration rates of individual components of the bidisperse mixture. The equations for calculating the maximum heat-transfer coefficient and the optimal filtration rate in the bidisperse mixture has been obtained.

Keywords: heat-exchange, fluidized bed, bidisperse bed, equivalent diameter, maximum heat-transfer coefficient, optimal filtration rate

For citation: Buchilko E. K. External heat-exchange in a quasi-bidisperse granular fluidized bed. Vestsi Natsyyanal'nai akademii navuk Belarusi. Seryya fizika-technichnych navuk = Proceedings of the National Academy of Sciences of Belarus. Physical-technical series, 2021, vol. 66, no. 2, pp. 220-226 (in Russian). https://doi.org/10.29235/1561-8358-2021-66-2-220-226

Введение. Техника псевдоожижения, как известно, получила широкое распространение в различных отраслях промышленности. Псевдоожиженный (кипящий) слой твердых частиц обладает такими уникальными свойствами, как высокие коэффициенты теплопроводности, высокая изотермичность слоя и практически идеальное перемешивание твердой фазы. Высокие коэффициенты теплообмена с погруженными поверхностями и стенками аппарата значительно упрощают организацию отвода и подвода тепла [1].

(C) Бучилко Э.К., 2021 
В последние годы появился интерес к использованию технологии псевдоожижения для термической переработки различных видов биоотходов (торрефикация, газификация, сжигание). В таких процессах кипящий слой зачастую представляет собой смесь двух полидисперсных сортов частиц, которые могут существенно отличаться формой, размерами и плотностью: частиц инерта размером порядка $0,2 \div 2,0$ мм и частиц перерабатываемого материала. Данный кипящий слой условно можно назвать квазибидисперсным. Смеси частиц разного размера и плотности особый класс зернистых слоев, гидродинамика и теплообмен в которых изучен недостаточно.

Цель настоящей работы - экспериментальное исследование внешнего теплообмена псевдоожиженного полидисперсного слоя с погруженным горизонтальным пучком труб при различных массовых соотношениях компонентов, составляющих слой.

Методика исследования. Исследование теплообмена полидисперсного слоя частиц с трубным пучком выполнено на экспериментальной установке, схема которой приведена на рис. 1. Основным элементом экспериментальной установки является прозрачная цилиндрическая камера псевдоожиженного (кипящего) слоя (КС) диаметром 172/180 мм, выполненная из

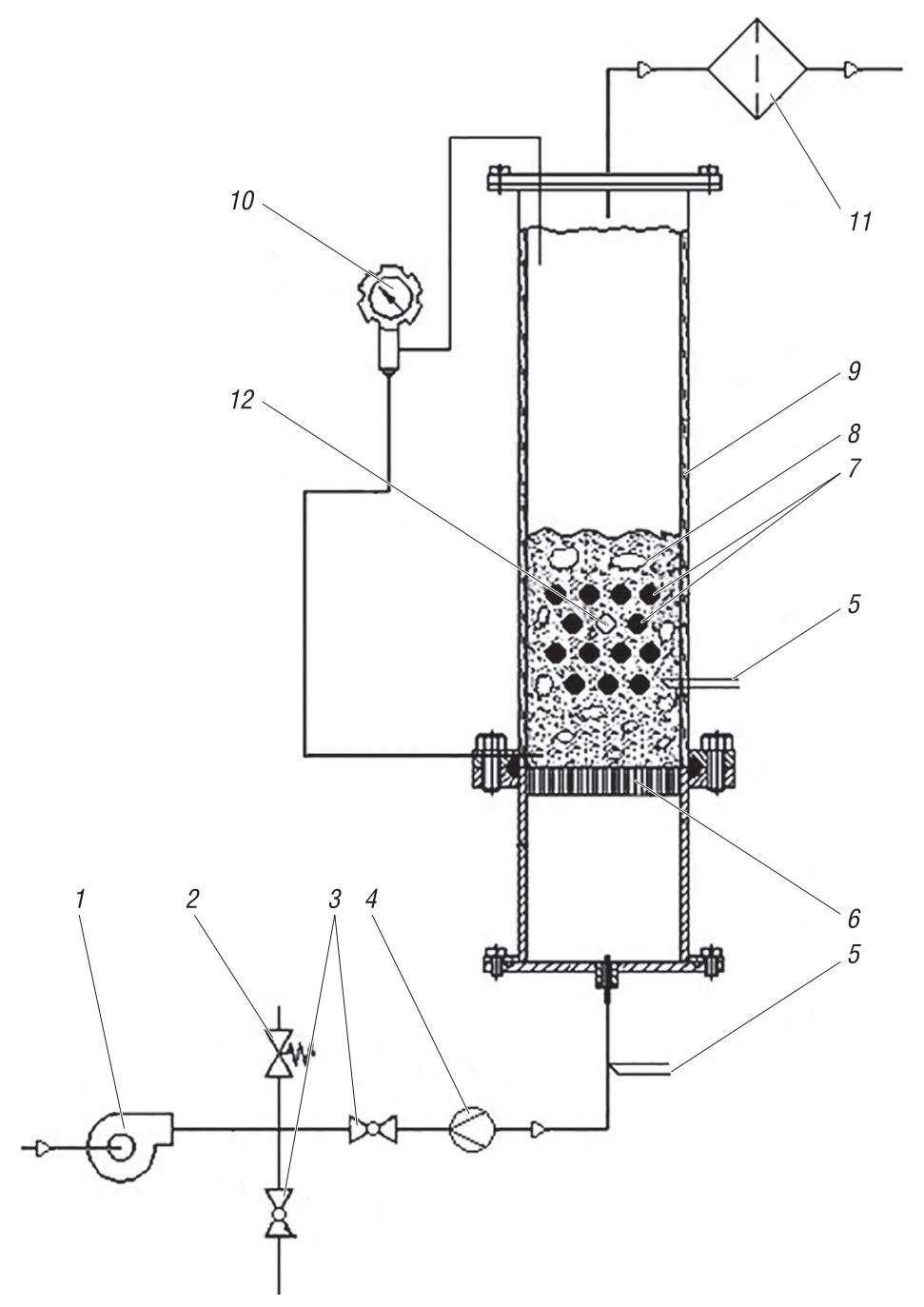

Рис. 1. Схема экспериментальной установки: 1 - воздуходувка, 2 клапан предохранительный, 3 - регулирующая арматура, 4 - расходомер, 5 - термопара, 6 - газораспределительная решетка, 7 - трубный пучок, 8 - кипящий слой, 9 - камера кипящего слоя, 10 - датчик перепада давления, 11 - фильтр, 12 - датчик теплообмена

Fig. 1. Scheme of the experimental setup: 1 - blower, 2 - relief valve, 3 - control valves, 4 - flow meter, 5 - thermocouple, 6 - gas distribution grid, 7 - tube bundle, 8 - fluidized bed, 9 - fluidized bed chamber, $10-$ differential pressure sensor, 11 - filter, 12 - heat-exchange sensor 
органического стекла. Высота камеры - 1,2 м. Газораспределительная решетка представляет собой спеченный порошок нержавеющей стали толщиной 10 мм и живым сечением $\approx 3 \%$.

В камеру КС экспериментального стенда была установлена кассета из горизонтальных цилиндрических элементов, имитирующих трубный пучок. Наружный диаметр цилиндрических элементов составлял 30 мм. Трубный пучок имел шахматную схему расположения элементов. Шаг труб $S_{\Gamma} \times S_{\mathrm{B}}=55 \times 50$ мм. Число горизонтальных рядов труб в пучке равнялось 4 . В третьем ряду пучка был установлен датчик теплообмена (рис. 2), представляющий собой медную трубку с внутренним нагревателем и подключенный к регулируемому источнику питания. Температура поверхности датчика измерялась хромель-копелевой термопарой. Для исключения торцевых потерь концы датчика были теплоизолированы фторопластовыми пробками.

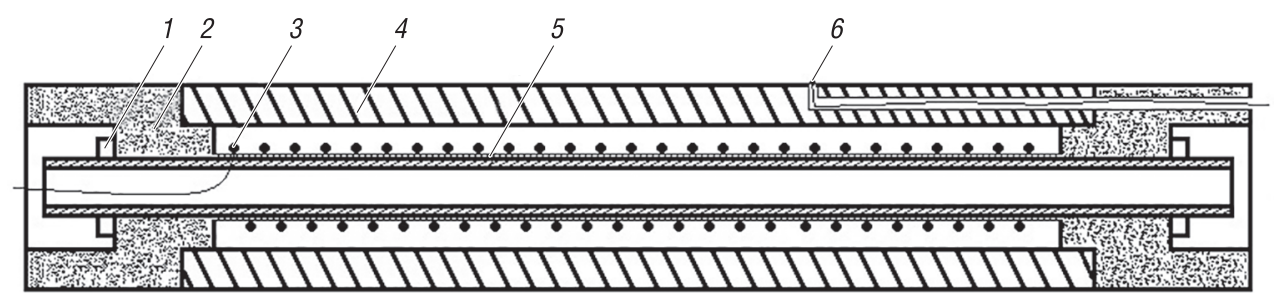

Рис. 2. Датчик теплообмена: 1 - гайка стягивающая, 2 - колпачок теплоизолирующий, 3 спираль электронагревателя, 4 - медный цилиндр, 5 - трубка из нержавеющей стали, 6 - королек термопары

Fig. 2. Heat-exchange sensor: 1 - tightening nut, 2 - heat-insulating cap, 3 - electric heater spiral, 4 - copper cylinder, 5 - stainless steel tube, 6 - thermocouple

Для исследования теплообмена между кипящим слоем и поверхностью использовался метод стационарного теплового потока, по которому коэффициент теплоотдачи определялся по формуле [2]

$$
\alpha=\frac{P}{F \cdot \Delta T}=\frac{U \cdot I}{F \cdot \Delta T},
$$

где $P$ - мощность, выделяемая нагревателем, Вт; $U$ - напряжение, $\mathrm{B} ; I$ - сила тока, А; $F$ - площадь теплоотдающей поверхности, $\mathrm{m}^{2} ; \Delta T$ - разность температур между поверхность датчика и псевдоожиженным слоем, ${ }^{\circ} \mathrm{C}$.

Для исследования теплоотдачи трубного пучка был выбран метод локального (местного) моделирования, который заключается в том, что в опытах участвует только одна из труб, расположенная в том месте, где производится исследование теплоотдачи [3].

В качестве объектов исследования были взяты смеси двух сортов полифракционных частиц: оливиновый - кварцевый пески (смесь I); оливиновый песок - пеллеты из соломы (смесь II). Доля крупных частиц в смеси ( () в экспериментах варьировалась от 0 до $100 \%$. Гистограммы распределений частиц в исследуемых полидисперсных слоях приведены на рис. 3.

Обсуждение результатов. На рис. 4, 5 представлены экспериментальные зависимости коэффициента теплоотдачи от скорости фильтрации в псевдоожиженном слое смесей I и II при разных массовых долях крупных частиц.

Как видно из рис. 4,5 , с ростом доли крупных частиц в смеси максимальный коэффициент теплоотдачи $\alpha_{\max }$ падает, что, очевидно, связано с ростом эквивалентного диаметра частиц слоя и подтверждается экспериментальными данными $[4,5]$.

В соответствии с уравнением (1) и правилом определения погрешностей косвенных измерений [2] относительная погрешность измерения коэффициентов теплоотдачи определялась по формуле

$$
\delta_{\alpha}=\sqrt{\left(\frac{\Delta I}{I}\right)^{2}+\left(\frac{\Delta U}{U}\right)^{2}+\left(\frac{\Delta T}{T}\right)^{2}+\left(\frac{\Delta F}{F}\right)^{2}},
$$

где $\Delta I, \Delta U, \Delta T, \Delta F$ - соответственно допустимая абсолютная погрешность измерения силы тока, напряжения, температурного напора, площади поверхности датчика, определяемая характеристиками измерительного прибора. 

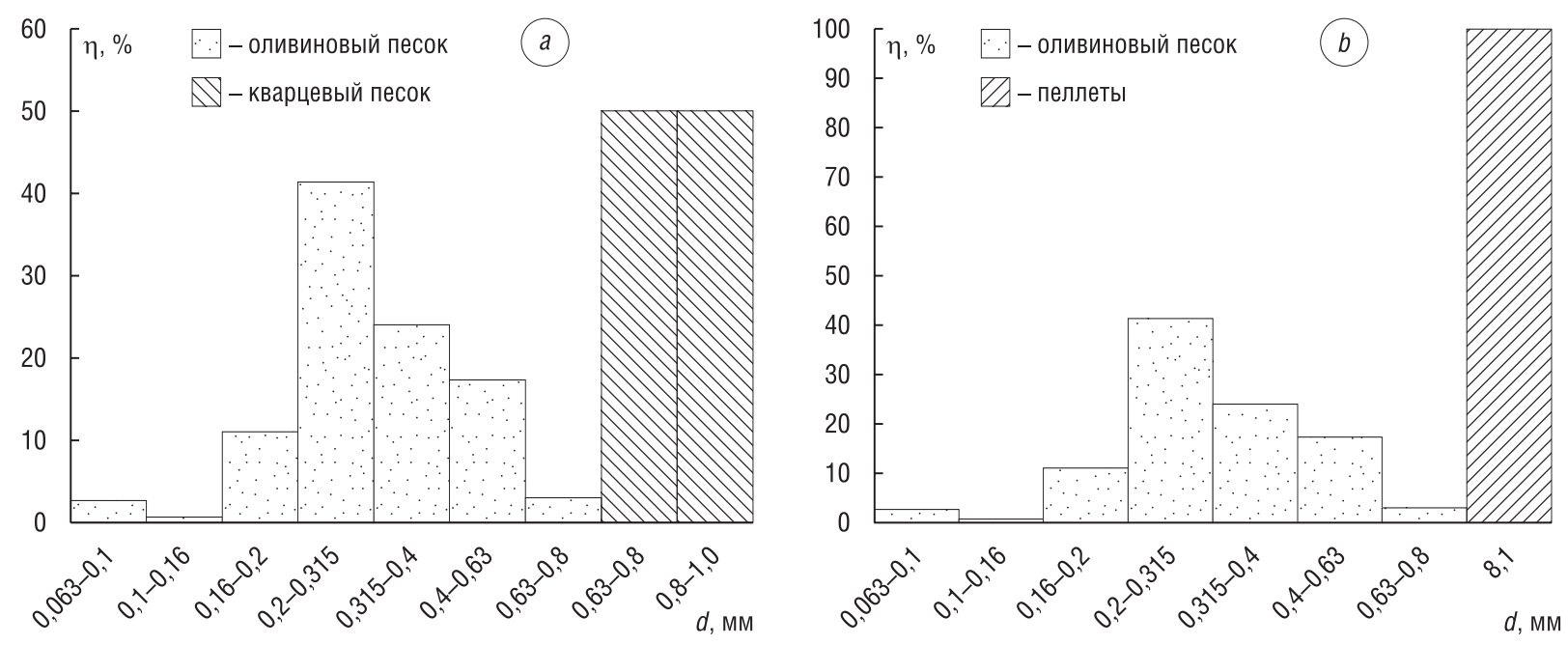

Рис. 3. Гистограммы распределений частиц исследуемых смесей: $a$ - оливиновый песок - кварцевый песок, $b-$ оливиновый песок - пеллеты

Fig. 3. Histograms of particle distributions of the studied mixtures: $a$ - olivine sand - quartz sand, $b$ - olivine sand - pellets

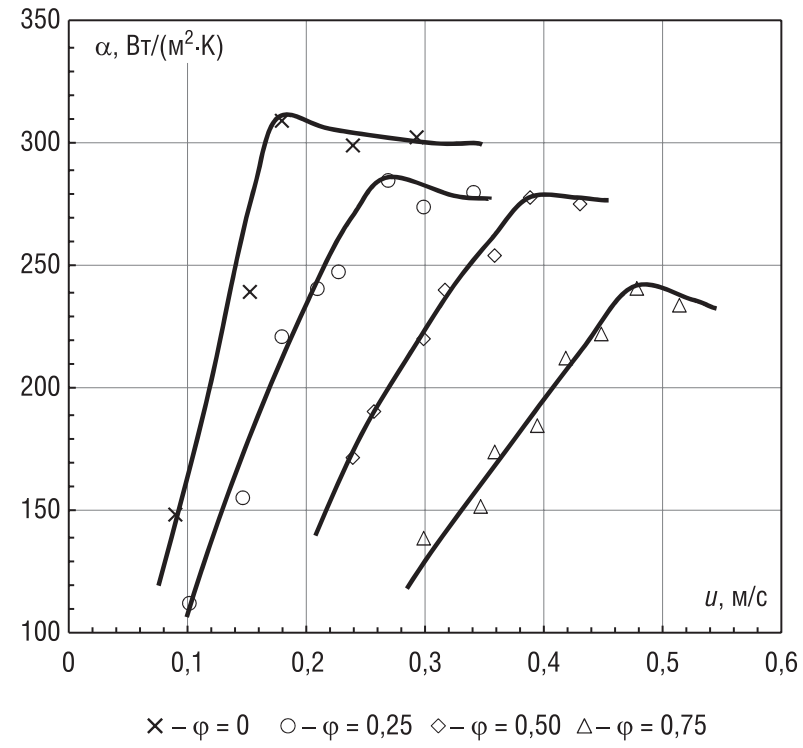

Рис. 4. Зависимость коэффициента теплоотдачи от скорости фильтрации для смеси I при разных массовых долях крупных частиц

Fig. 4. Dependence of the heat transfer coefficient on the filtration rate for mixture $I$ at different mass fractions of large particles

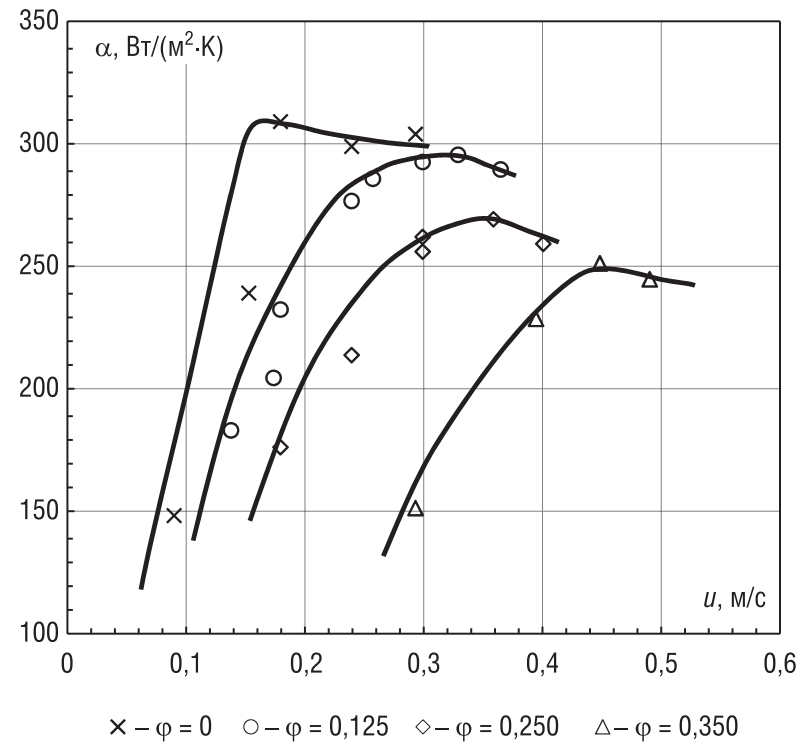

Рис. 5. Зависимость коэффициента теплоотдачи от скорости фильтрации для смеси II при разных массовых долях крупных частиц

Fig. 5. Dependence of the heat transfer coefficient on the filtration rate for mixture II at different mass fractions of large particles

В результате максимальная относительная погрешность измерения коэффициента теплоотдачи в псевдоожиженном квазибидисперсном слое составила не более 8 \%.

Сложность расчета теплоотдачи в псевдоожиженном слое полидисперсных смесей с широким фракционным составом заключается в необходимости выбора эквивалентного диаметра частиц. Существуют разные способы нахождения эквивалентного диаметра, среди которых наибольшее распространение получил метод усреднения различных степеней диаметров частиц отдельных фракций [1]:

$$
d_{\mathrm{m}}^{\mathrm{K}}=\sum \eta_{i} d_{i}^{\mathrm{K}} .
$$

При $\mathrm{K}=1$ определяется средневесовой диаметр, а при $\mathrm{K}=-1$ - среднеповерхностный диаметр. Применительно к исследуемым квазибидисперсным системам формула (3) примет вид: 


$$
\begin{aligned}
& d_{\mathrm{m}}=d_{1}=\varphi\left(\sum_{i=1}^{N^{(1)}} d_{i}^{(1)} \eta_{i}^{(1)}\right)+(1-\varphi)\left(\sum_{i=1}^{N^{(2)}} d_{i}^{(2)} \eta_{i}^{(2)}\right)-\text { средневесовой диаметр; } \\
& d_{\mathrm{m}}=d_{2}=\frac{1}{\left(\varphi \sum_{i=1}^{N^{(1)}} \frac{\eta_{i}^{(1)}}{d_{i}^{(1)}}+(1-\varphi) \sum_{i=1}^{N^{(2)}} \frac{\eta_{i}^{(2)}}{d_{i}^{(2)}}\right)}-\text { среднеповерхностный диаметр. }
\end{aligned}
$$

В [6] установлено, что средневесовой диаметр в качестве эквивалентного дает существенно заниженные значения коэффициента теплоотдачи и предпочтительно использовать среднеповерхностный диаметр. Анализ полученных экспериментальных данных также показал, что использование (5) для расчета эквивалентного диаметра лучше описывает опытные данные.

Для обобщения полученных опытных данных использовался метод обобщения с помощью максимальных коэффициентов теплоотдачи отдельных составляющих смеси $\alpha_{\max }^{\mathrm{I}}$ и $\alpha_{\max }^{\mathrm{II}}$, которые в большинстве случаев можно рассчитать по одной из известных эмпирических формул $[1,5,6]$. При этом полученные данные были обобщены в виде двух простых уравнений:

$$
\begin{gathered}
\left(\alpha_{\max }\right)_{\operatorname{mix}}=\varphi \cdot \alpha_{\max }^{\mathrm{I}}+(1-\varphi) \cdot \alpha_{\max }^{\mathrm{II}}, \\
\frac{1}{\left(\alpha_{\max }\right)_{\operatorname{mix}}}=\frac{\varphi}{\alpha_{\max }^{\mathrm{I}}}+\frac{1-\varphi}{\alpha_{\max }^{\mathrm{II}}} .
\end{gathered}
$$

Аналогичным образом были обобщены опытные данные по оптимальным (по теплообмену)

$\lg \mathrm{Re}$

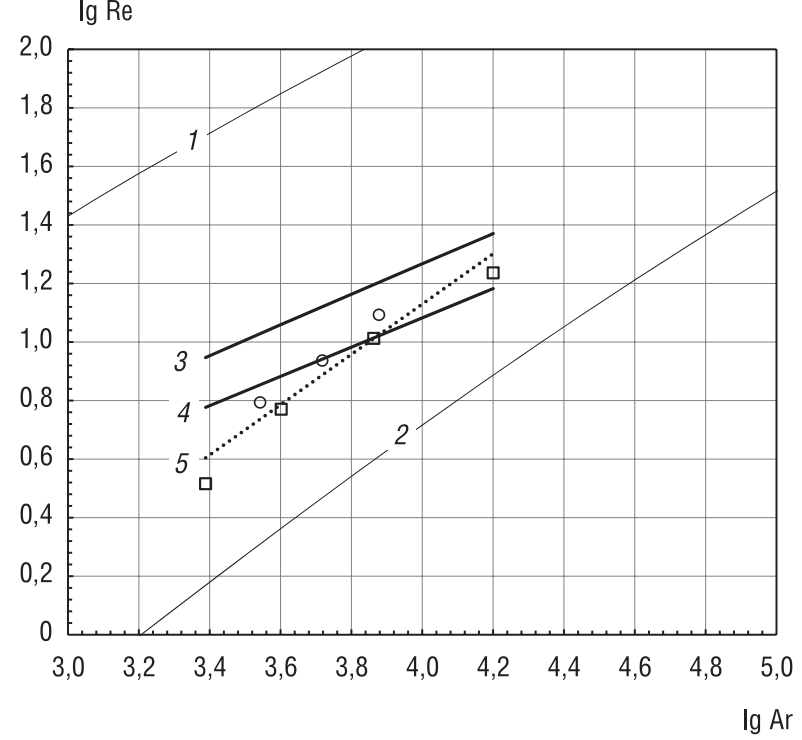

Рис. 6. Зависимость положения максимума коэффициента теплоотдачи от критерия Архимеда:

$$
\begin{gathered}
1-\operatorname{Re}_{\mathrm{t}}=\frac{\mathrm{Ar}}{18+0,61 \sqrt{\mathrm{Ar}}}[1] ; 2-\mathrm{Re}_{\mathrm{mf}}=\frac{\mathrm{Ar}}{1400+5,22 \sqrt{\mathrm{Ar}}}[1] ; \\
3-\mathrm{Re}_{\mathrm{opt}}=\frac{\mathrm{Ar}}{18+5,22 \sqrt{\mathrm{Ar}}}[1] ; 4-\mathrm{Re}_{\mathrm{opt}}=0,121 \mathrm{Ar}^{0,5}[1] ; \\
5-\mathrm{Re}_{\mathrm{opt}}=0,004 \mathrm{Ar}^{0,88} \text { (экспериментальные данные) }
\end{gathered}
$$

Fig. 6. Dependence of the maximum heat transfer coefficient

$$
\begin{gathered}
\text { position on the Archimedes criterion: } \\
1-\mathrm{Re}_{\mathrm{t}}=\frac{\mathrm{Ar}}{18+0,61 \sqrt{\mathrm{Ar}}}[1] ; 2-\mathrm{Re}_{\mathrm{mf}}=\frac{\mathrm{Ar}}{1400+5,22 \sqrt{\mathrm{Ar}}}[1] \\
3-\mathrm{Re}_{\mathrm{opt}}=\frac{\mathrm{Ar}}{18+5,22 \sqrt{\mathrm{Ar}}}[1] ; 4-\mathrm{Re}_{\mathrm{opt}}=0,121 \mathrm{Ar}^{0,5}[1] \\
5-\mathrm{Re}_{\mathrm{opt}}=0,004 \mathrm{Ar}^{0,88} \text { (experimental data) }
\end{gathered}
$$
скоростям фильтрации исследуемых смесей:

$$
\begin{gathered}
\left(u_{\mathrm{opt}}\right)_{\text {mix }}=\varphi \cdot u_{\mathrm{opt}}^{\mathrm{I}}+(1-\varphi) \cdot u_{\mathrm{opt}}^{\mathrm{II}}, \\
\frac{1}{\left(u_{\mathrm{opt}}\right)_{\operatorname{mix}}}=\frac{\varphi}{u_{\mathrm{opt}}^{\mathrm{I}}}+\frac{1-\varphi}{u_{\mathrm{opt}}^{\mathrm{II}}} .
\end{gathered}
$$

Для нахождения оптимальной скорости фильтрации отдельных компонентов, составляющих смесь, на основе опытных данных (рис. 6) была получена интерполяционная зависимость вида

$$
\mathrm{Re}_{\text {opt }}=0,004 \mathrm{Ar}^{0,88} \text {. }
$$

Для сравнения на рис. 6 показаны зависимости максимума коэффициента теплоотдачи от критерия Архимеда для рассматриваемых бидисперсных смесей частиц, которые были рассчитаны по другим известным формулам.

В таблице приведены измеренные величины максимального коэффициента теплоотдачи $\left(\alpha_{\max }\right)_{\text {эксп }}$, оптимальной скорости фильтрации

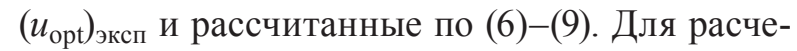
та коэффициента теплоотдачи отдельных составляющих смеси выбрана формула, предложенная Баскаковым [5]:

$$
\mathrm{Nu}_{\max }=0,85 \operatorname{Ar}^{0,19}+0,006 \operatorname{Ar}^{0,5} \operatorname{Pr}^{0,33}
$$

Другие распространенные зависимости для нахождения коэффициента теплоотдачи 
к погруженным в слой телам (формула Забродского [7], Варыгина-Матюшина [1]) дают сопоставимые с (11) результаты.

\section{Расчетные и экспериментальные данные для исследуемых смесей}

Calculated and experimental data for the mixtures under study

\begin{tabular}{|c|c|c|c|c|c|c|c|c|c|}
\hline Смесь & $\varphi$ & $d_{\mathrm{m}}$ по (5), мм & $\begin{array}{c}\alpha_{\max } \text { по (11), } \\
\mathrm{BT} /\left(\mathrm{M}^{2} \cdot \mathrm{K}\right)\end{array}$ & $\begin{array}{l}\left(\alpha_{\max }\right)_{\text {эксп }} \\
\mathrm{BT} /\left(\mathrm{M}^{2} \cdot \mathrm{K}\right)\end{array}$ & $\left(u_{\text {opt }}\right)_{\text {эксп }}, \mathrm{M} / \mathrm{c}$ & $\begin{array}{c}\alpha_{\max } \text { по (6), } \\
\mathrm{BT} /\left(\mathrm{M}^{2} \cdot \mathrm{K}\right)\end{array}$ & $\begin{array}{c}\alpha_{\max } \text { по (7), } \\
\mathrm{BT} /\left(\mathrm{M}^{2} \cdot \mathrm{K}\right)\end{array}$ & $u_{\text {opt }}$ по (8), м/с & $u_{\text {opt }}$ по (9),, $\mathrm{M} / \mathrm{c}$ \\
\hline \multirow[t]{5}{*}{ I } & 0 & 0,27 & 378 & 310 & 0,18 & 378 & 378 & 0,21 & 0,21 \\
\hline & 0,25 & 0,33 & 351 & 285 & 0,27 & 346 & 336 & 0,40 & 0,26 \\
\hline & 0,50 & 0,41 & 321 & 278 & 0,38 & 314 & 301 & 0,60 & 0,35 \\
\hline & 0,75 & 0,54 & 288 & 241 & 0,48 & 282 & 274 & 0,79 & 0,51 \\
\hline & 1,0 & 0,80 & 251 & - & - & 251 & 251 & 0,98 & 0,98 \\
\hline \multirow[t]{5}{*}{ II } & 0 & 0,27 & 378 & 310 & 0,18 & 378 & 378 & 0,21 & 0,21 \\
\hline & 0,125 & 0,31 & 358 & 292 & 0,30 & 350 & 318 & 3,05 & 0,25 \\
\hline & 0,250 & 0,36 & 337 & 270 & 0,36 & 322 & 275 & 5,89 & 0,29 \\
\hline & 0,350 & 0,42 & 318 & 250 & 0,45 & 299 & 249 & 8,16 & 0,32 \\
\hline & 1,0 & 8,10 & 152 & - & - & 152 & 152 & 22,90 & 22,90 \\
\hline
\end{tabular}

Из таблицы видно, что экспериментальные и расчетные величины коэффициента теплоотдачи лучше согласуются при расчете по формуле (7), чем (6). Особенно это характерно для смеси II $\left(\frac{d_{\mathrm{I}}}{d_{\mathrm{II}}} \geqslant 20\right)$. Расчетные значения оптимальной скорости фильтрации также лучше соответствуют экспериментальным при использовании для ее расчета зависимости вида (9). В этом случае лучшее совпадение показывает смесь I $\left(\frac{d_{\mathrm{I}}}{d_{\mathrm{II}}}=2,5\right)$.

Заключение. Разработан метод расчета максимального коэффициента теплоотдачи и оптимальной скорости фильтрации для зернистого слоя двух сортов полидисперсных частиц, которые могут существенно отличаться формой, размерами и плотностью. При расчете теплоотдачи в таких полидисперсных слоях рекомендовано использовать среднеповерхностный диаметр в качестве эквивалентного. Для нахождения оптимальной скорости фильтрации отдельных составляющих, рассматриваемых бидисперсных смесей, предложена интерполяционная зависимость (10).

Полученные зависимости для расчета максимального коэффициента теплоотдачи (7) и оптимальной скорости фильтрации (9) позволяют удовлетворительно описать опытные данные.

\section{Благодарности}

Работа выполнена при финансовой поддержке Белорусского республиканского фонда фундаментальных исследований (проект Т19РМ-009).

\section{Acknowledgments}

This work was carried out with financial support of the Belarusian Republican Foundation for Fundamental Research (project No. T19PM-009).

\section{Обозначения, принятые в статье}

$\operatorname{Ar}=\frac{\rho_{\mathrm{f}} g d_{\mathrm{s}}^{3}}{\mu_{\mathrm{f}}^{2}}\left(\rho_{\mathrm{s}}-\rho_{\mathrm{f}}\right)-$ число Архимеда; $\operatorname{Re}=\frac{u d_{\mathrm{s}}}{v_{\mathrm{f}}}-$ число Рейнольдса; $\mu-$ динамическая вязкость газа, кг/(м·c); $v-$ кинематическая вязкость газа, $\mathrm{m}^{2} / \mathrm{c} ; d_{\mathrm{m}}$ - эквивалентный диаметр смеси, мм; $d_{1}$ - средневесовой диаметр смеси, м; $d_{2}$ - среднеповерхностный диаметр смеси, м; $d_{\mathrm{I}}-$ средневесовой диаметр крупных частиц, м; $d_{\mathrm{II}}-$ средневесовой диаметр мелких частиц, м; $d_{i}^{(1)}-$ диаметр частиц $i$-й фракции первого материала бидисперсной смеси, м; $d_{i}^{(2)}-$ диаметр частиц $i$-й фракции второго материала бидисперсной смеси, м; $\eta_{i}$ - массовая доля $i$-й фракции; $\varphi-$ массовая доля крупных частиц бидисперсной смеси; $u$ - скорость фильтрации газа, м/с; $\alpha-$ коэффициент теплоотдачи, Вт/(м²·К).

Индексы: $\mathrm{s}$ - частицы; $\mathrm{f}$ - газ; $\mathrm{t}$ - витание; $\mathrm{mf}$ - начало псевдоожижения; mix - смесь частиц; орt - оптимальный; max - максимальный; эксп - экспериментальный. 


\section{Список использованных источников}

1. Аэров, М.Э. Гидравлические и тепловые основы работы аппаратов со стационарным и кипящим зернистым слоем / М.Э. Аэров, О. М. Тодес. - Л.: Химия, 1968. - 512 с.

2. Осипова, В. А. Экспериментальное исследование процессов теплообмена. - М.: Энергия, 1979. - 320 с.

3. Исаченко, В.П. Теплопередача / В. П. Исаченко, В. А. Осипова, А. С. Сукомел. - М.: Энергия, 1975. - 488 с.

4. Гельперин, Н.И. Основы техники псевдоожижения / Н.И. Гельперин, В.Г. Айнштейн, В.Б. Кваша. - М.: Химия, 1967. -664 с.

5. Баскаков, А.П. Котлы и топки с кипящим слоем / А.П. Баскаков, В.В. Мацнев, И.В. Распопов. - М.: Энергоатомиздат, 1996. - 352 с.

6. Теплицкий, Ю.С. Гидродинамика и тепломассоперенос в свободном и заторможенном псевдоожиженных слоях: автореф. дис. ... д-ра техн. наук: 01.04.14 / Ю. С. Теплицкий; Ин-т теплофизики СО АН СССР. - Новосибирск, 1990. $-65 \mathrm{c}$.

7. Забродский, С.С. Гидродинамика и теплообмен в псевдоожиженном (кипящем) слое. - М.; Л.: Госэнергоиздат, 1963. $-488 \mathrm{c}$.

\section{References}

1. Aerov M. E., Todes O.M. Hydraulic and Thermal Principles of Operation of Apparatuses with Fixed and Fluidized Granular Bed. Leningrad, Khimiya Publ., 1968. 510 p. (in Russian).

2. Osipova V. A. Experimental Study Processes of Heat Transfer. Moscow, Energia Publ., 1979. 488 p. (in Russian).

3. Isachenko, V. P., Osipova, V. A. Sukomel', A. S. Heat Transfer. Moscow, Energoizdat Publ., 1981. 416 p. (in Russian).

4. Gel'perin N. I., Ainshtein V. G., Kvasha V.B. Fundamentals of Fluidization Techniques. Moscow, Khimiya Publ., 1967. 664 p. (in Russian).

5. Baskakov A. P., Matsnev I. V. Boilers and Furnaces with a Fluidized Bed. Moscow, Energoatomizdat Publ., 1996. 352 p. (in Russian).

6. Teplickij Yu. S. Hydrodynamics and Heatmass Transfer in Free and Delayed Fluidized Beds. Novosibirsk, 1990. 65 p. (in Russian).

7. Zabrodskii S. S. Hydrodynamics and Heat Transfer in Fluidized Bed. Moscow-Leningrad, Gosenergoizdat Publ., 1963. 488 p. (in Russian).

\section{Информация об авторе}

Бучилко Эдуард Казимирович - научный сотрудник, Институт тепло- и массообмена имени А.В. Лыкова Национальной академии наук Беларуси (ул. П. Бровки, 15, 220072, Минск, Республика Беларусь). E-mail: ebuchilko@gmail.com

\section{Information about the author}

Eduard K. Buchilko - Researcher, A. V. Luikov Heat and Mass Transfer Institute of the National Academy of Sciences of Belarus (15, Brovka Str., 220072, Minsk, Republic of Belarus).E-mail: ebuchilko@gmail.com 